\title{
Lexicographic Maximin Optimisation for Fair Bandwidth Allocation in Computer Networks
}

\author{
Ronaldo M. Salles* \\ Javier A. Barria ${ }^{\dagger}$ \\ (corresponding author)
}

\begin{abstract}
This paper addresses the problem of bandwidth allocation in multi-application computer network environments. Allocations are determined from the solution of a multiple objective optimisation problem under network constraints, where the lexicographic maximin criterion is applied to solve the problem and guarantees fairness and efficiency properties to the solution. An algorithm based on a series of maximum concurrent multicommodity flow sub-problems is proposed. Numerical results show the advantage of the approach compared to other traditional bandwidth allocation solutions.
\end{abstract}

Keywords: OR in telecommunications, bandwidth allocation, lexicographic optimisation, fairness, utility theory.

\section{Introduction}

The performance of network applications is directly affected by the amount of available bandwidth along end-to-end paths. Optimizing the allocation of bandwidth on network links is therefore a fundamental issue toward the improvement of network services.

In the network context, it is often possible to map the amount of allotted bandwidth to the expected level of performance experienced by the application. From microeconomic theory (Mas-Colell et al. 1995), such mapping is known as the utility function associated to the application, or simply, application utility.

Utility functions can be determined either qualitatively, through typical application behaviour (Shenker, 1995), or quantitatively, through mean opinion scores (MOS), distortion rates (Berger, 1971) or closed form expressions (Breslau and Shenker, 1998; Liao and Campbell 2001; Salles and Barria 2004). From the user point of view, her personal

\footnotetext{
*Dept. de Eng. de Sistemas, Instituto Militar de Engenharia, Praia Vermelha 80, 22290-270, Rio de Janeiro, Brazil, Phone: +55-21-2546-7120(7090), FAX: +55-21-2546-7099 (salles@ieee.org).

${ }^{\dagger}$ Dept. of Electrical and Electronic Engineering, Imperial College London, Exhibition Road, SW7 2BT, London, U.K. (j.barria@imperial.ac.uk).
} 
goal may be to maximise utility. In this sense, the bandwidth allocation problem can be generally formulated as a constrained multiple objective optimisation problem (CMOP): multiple utility functions to be maximised under constraints given by the limited amount of resources (link bandwidth) distributed according to the network topology.

The most traditional solution for bandwidth allocation problems is the max-min fair solution (Bertsekas and Gallager, 1992). However, it has been argued that it may generate sub-optimal allocations in several contexts depending on the actual utility function of the applications (Gevros et al. 2001). On the other hand, Kelly et al. (1998) introduced the idea of proportional fairness where each application $j$ is associated with a strictly concave utility function $u(x, j)$ (logarithmic) and allocations $x$ are determined from the solution of $\max _{x} \sum_{j=1}^{N} u(x, j)$. However, if utility functions are not all concave, proportional fair allocations are no more unique (several local solutions may exist) and may be completely unbalanced: some applications may experience zero utilities while others experience a very good performance.

In this paper we formulate the bandwidth allocation problem as a general CMOP and allow utility to be any continuous and monotone function to reflect the diversity of applications observed in network environments today. We adopt the lexicographic maximin criterion as a way to mediate conflicts in the CMOP since it provides solutions satisfying fairness and efficiency properties. We propose an algorithm based on a sequence of maximum concurrent multicommodity flow subproblems and compare the solutions returned by it with solutions from traditional approaches.

This work is organized as follows. Section 2 formulates the problem and presents decision criteria together with their properties. Section 3 presents some related work on the topic. In Section 4 the lexicographic maximin solution is studied and a preliminary procedure is presented. Section 5 proposes the main algorithm followed by some numerical results in Section 6. Section 7 discusses limitations, considerations and practical issues related to the proposed approach. Finally, Section 8 concludes the work.

\section{Problem Formulation}

The problem of bandwidth allocation in multi-application network environments can be addressed using the framework of Social Choice Theory and Welfare Economics since it constitutes a form of division problem (Sen, 1995). In this case, a set of applications contend for the distributed network resources and among all feasible allocations, one of them is selected as the best alternative.

Each application is associated to a utility function that represents the performance perceived by end-users when using the allotted network resources. Traffic generated from 
applications is assumed to be a continuous flow of information that follows network paths from origin to destination consuming all the allotted resources and being split over the available paths if necessary. In this sense, applications are associated to monotone utility functions: the more resources are allocated to the application the higher is the utility. Several continuous and monotone function are used to describe that relationship - e.g. concave, convex, s-shaped, linear, etc. Such approach covers a large variety of applications.

Let the network topology be given by a graph $\mathcal{G}:(\mathcal{V}, \mathcal{L})$, where $\mathcal{V}$ is the set of nodes, and $\mathcal{L}$ the set of links along with each link $l$ capacity $C_{l}$. Each application generates network traffic from one origin node to another destination node - a commodity flow, with $\mathcal{N}$ as the set of all commodities. Let $P_{j}$ be the set of directed paths available for commodity $j \in \mathcal{N}: P_{j}=\left\{p_{j 1}, p_{j 2}, \ldots, p_{j\left|P_{j}\right|}\right\}$. Let $x_{j k}$ be the flow on the $k$-th path associated to commodity $j$, and $\boldsymbol{x}$ the vector of all path commodity flows. We define $u(\boldsymbol{x}, j)$ as the utility function associated to $j \in \mathcal{N}$.

The set of feasible allocations $\mathcal{X}$ (domain set) must satisfy all network capacity constraints and non-negativity conditions. Using the notation above, it is given by

$$
\mathcal{X}=\left\{\sum_{j \in \mathcal{N}} \sum_{p_{j k} \in P_{j}} \delta_{l}\left(p_{j k}\right) x_{j k} \leq C_{l} ; \quad x_{j k} \geq 0 ; \quad l \in \mathcal{L}\right\}
$$

where $\delta_{l}\left(p_{j k}\right)$ is the indicator function (=1 if path $p_{j k}$ uses link $l,=0$ otherwise)

Since applications are engaged in maximizing their own utility functions, the bandwidth allocation problem reduces to a constrained multiple objective optimisation problem (CMOP) given below in a compact form,

$$
\max _{\substack{\boldsymbol{x} \in \mathcal{X} \\ \forall j \in \mathcal{N}}}\{u(\boldsymbol{x}, j)\}
$$

However, CMOPs like (2) are generally ill-defined (Miettinen, 1998) due to the interrelation observed in the objective functions - if we try to maximise $u(\boldsymbol{x}, i)$ we may end up minimizing $u(\boldsymbol{x}, k)$, and so there is a need to select a criterion to carry out the optimisation.

\subsection{Decision Criteria}

An important condition the solution should satisfy is Pareto Efficiency. This condition has been widely applied in Economics (Mas-Colell et al. 1995), Social Choice Theory (Sen, 1995), Game Theory (Luce and Raiffa, 1989), and Multiple Objective Optimisation (Miettinen, 1998). In general terms, an allocation of resources is Pareto Efficient if no agent can improve his own utility without decreasing the utility of others. A Pareto 
Efficient allocation is said to be non-dominated by any other allocation. For instance, if the utilitarian criterion (overall sum of utilities) is applied to our problem we have,

$$
\max _{x \in \mathcal{X}} \sum_{j \in \mathcal{N}} \boldsymbol{u}(x, j)
$$

where the optimal solution $\boldsymbol{x}^{*}$ is Pareto Efficient.

Criticism on the Pareto Criterion dates back 1971 from Rawls' egalitarian principle. The main concern is that there is no fairness component embedded in the criterion. An allocation where agent $\mathcal{A}$ gets all the resources while agent $\mathcal{B}$ gets nothing may still be Pareto Efficient. In fact, if each agent is engaged in maximizing his own benefit, fairness tends to disappear.

Another commonly employed approach to sort out multiple objective problems as in (2) is to use lexicographic ordering (Miettinen, 1998). A natural example of lexicographic ordering is the way words are sorted in the dictionary. Mathematically speaking, it is the ordinance of the cartesian product of two sets A and the B. Given A and B, two ordered sets, the lexicographical order in the Cartesian product $A \times B$ is defined as:

$$
\begin{aligned}
& \text { If }\left(a_{1}, b_{1}\right) \text { and }\left(a_{2}, b_{2}\right) \text { belongs to } A \times B \text {, so }\left(a_{1}, b_{1}\right)<\left(a_{2}, b_{2}\right) \text { if and only if: } \\
& \qquad \begin{array}{l}
a_{1}<a_{2} \text {, or } \\
a_{1}=a_{2} \text { and } b_{1}<b_{2}
\end{array}
\end{aligned}
$$

Generalizing for a character sequence $A=\left(a_{1}, a_{2}, \ldots, a_{k}\right)$ and $B=\left(b_{1}, b_{2}, \ldots, b_{k}\right)$, $A<B$ if and only if the first $a_{i}$ which is different from $b_{i}$ must be lesser than $b_{i}$. In other words, a word $A$ will appear before a word $B$ if the letter $a_{i}$ appears before $b_{i}$ in the alphabet.

Regarding our problem, we are interested in real valued utility functions instead of letters in the alphabet. Hence, to employ the lexicographic ordering it is mandatory to make a priori arrangement of the utility functions according to their absolute importance. After ordering, the most important utility function is maximised subject to the original constraints. Then, from this solution, the second most important utility function is maximised and so on, until a unique solution is found.

Although the lexicographic procedure described above leads to a Pareto Efficient solution (Miettinen, 1998), this solution is not fair (egalitarian) since it is first necessary to establish a strict precedence among all utility functions. Therefore, this simple lexicographic approach is also not applicable to our problem.

Among the several notions of fairness, the egalitarian principle is probably the one which has been more extensively considered. It is usually defined through the Rawlsian 
criterion (Rawls, 1999): "the system is no better-off than its worse-off individual". Using this principle, fairness implies the maximization of the benefit (utility) of the worse-off individual. If we apply the egalitarian criterion to our problem we now have,

$$
\max _{x \in \mathcal{X}} \min _{j \in \mathcal{N}}\{\boldsymbol{u}(x, j)\}
$$

Although (4) guarantees fairness, efficiency (Pareto Efficiency) is usually not achieved. In fact, the three criteria studied (utilitarian, lexicographic and Rawlsian) illustrate the wellknown trade-off between fairness and efficiency which has been the subject of extensive research for many years (Sen, 1999).

However, one may note that the lexicographic extension of the Rawlsian criterion (here called lexicographic maximin) investigated in (Roberts, 1980) and in (Chen, 2000) under the name of leximin, is indeed Pareto Efficient. In the lexicographic maximin approach, a Rawlsian problem (4) is solved at each stage without wasting the previous stage solution until a Pareto Efficient point is found. In this way, the solution for the bandwidth allocation problem enjoys both fairness and efficiency proprieties, hence, the lexicographic maximin criterion is adopted in this paper and will be further studied.

\section{Related Work}

There has been several works in the literature that employed some type of fairness criteria to sort out network allocation problems.

The well-established bandwidth allocation concept of max-min fairness (Bertsekas and Gallager, 1992) has been investigated to a large extent and several different algorithms have been proposed so far. To cite just a few (Hahne, 1991), (Awerbuch and Shavitt, 1998), (Tsai and Kim, 1999) and (Tomaszewski, 2005). Max-min fairness deals with homogeneous commodity flows and leads to linear optimisation problems, and so it can not be applied to nonlinear and heterogeneous scenarios where commodity flows are specified by different types of utility functions.

Some works have also extended max-min fairness to consider utility function information, e.g. (Cao and Zegura, 1999) and (Lee and Chong, 2005). However, they are based on bottleneck theory and so they consider only fixed and single paths for each commodity flow. The problem discussed in this paper is nonlinear and multipath, hence, it represents a general formulation. The proposed algorithm can be applied to other simplified problems as well.

It should be noted that the criterion used in this work is in fact an extension of the Rawlsian criterion, also known as lexicographic maximin, and so it differs from lexico- 
graphic schemes generally applied to solve multiple objective optimisation problems as in (Pourkarimi and Zarepisheh, 2006) and (Volgenant, 2002). It was seen in Sec. 2.1 that the criterion is based on utility function information and has the advantage to provide not only fairness but also efficiency to the solution.

The work in (Ogryczak, 2004) presents an excelent discussion on bandwidth allocation criteria and provides a thorough review about max-min, proportional and lexicographic maximin allocations. They also highlight the importance of fairness and efficiency, and introduce new methods to generate fair efficient bandwidth allocations - the reference point procedure.

To the best of our knowledge our work presents for the first time a solution for the general nonlinear lexicographic maximin problem based only on linear MCMF (maximum concurrent multicommodity flow) subproblems. Next section studies the lexicographic maximin criterion in greater detail.

\section{The Lexicographic Maximin Solution}

Under the lexicographic maximin criterion whenever it is possible to increase the utility of a given agent without affecting any other worse-off agent, that agent should be made better-off. It is possible to mathematically characterize the lexicographic maximin solution from the social welfare funcionals (SWFL) studied in (Roberts, 1980, p.432). According to that work, the following SWFL: $\sum_{i \in N} \frac{[u(\boldsymbol{x}, i)]^{1-\rho}}{1-\rho}$ must be the lexicographic extension to the Rawlsian criterion when parameter $\rho \rightarrow \infty$. Thus, the lexicographic maximin solution can be obtained from the solution of the following optimisation problem:

$$
\boldsymbol{x}^{l e x}=\arg \max _{\boldsymbol{x} \in \mathcal{X}}\left\{\lim _{\rho \rightarrow \infty} \sum_{i \in N} \frac{[u(\boldsymbol{x}, i)]^{1-\rho}}{1-\rho}\right\}
$$

The limit in (5) makes the use of traditional optimisation procedures such as the method of Lagrangian multipliers not directly applicable to compute $\boldsymbol{x}^{\text {lex }}$. In fact, a better way to characterize this point happens to be in terms of the objective set (image of the domain set $\mathcal{X}$ ) and preference relations (Mas-Colell et al. 1995).

Let $\mathcal{Z} \subseteq \Re^{N}$ defines the objective set if it contains all points $\boldsymbol{z}:\left(z_{1}, z_{2}, \ldots, z_{N}\right)^{T}$, where $N$ is the dimension of set $\mathcal{N}, z_{j}=u(\boldsymbol{x}, j), \boldsymbol{x} \in \mathcal{X}$ and $j \in \mathcal{N}$. We want to characterise $\boldsymbol{z}^{l e x} \in \mathcal{Z}$ so that its components are given by $u\left(\boldsymbol{x}^{l e x}, j\right)$. Let $\pi$ be a permutation for any $\boldsymbol{z} \in \mathcal{Z}$, such that $\pi(\boldsymbol{z})=\boldsymbol{z}^{p}$ if,

$$
\begin{aligned}
z_{j}^{p} & \geq z_{j-1}^{p} \\
j & \in \mathcal{N}
\end{aligned}
$$


Thus, $\pi(\boldsymbol{z})$ simply puts the components of $\boldsymbol{z}$ in a nondecreasing order. ${ }^{1}$ We also define the preference operator $\succ$ over $\mathcal{Z}$, such that for any two feasible objective vectors $\boldsymbol{y}, \boldsymbol{z} \in \mathcal{Z}$ and permutations $\boldsymbol{y}^{p}=\pi(\boldsymbol{y}), \boldsymbol{z}^{p}=\pi(\boldsymbol{z}), \boldsymbol{y} \succ \boldsymbol{z}$ if $\exists m, 1 \leq m \leq N$, satisfying:

$$
\begin{aligned}
y_{j}^{p} & =z_{j}^{p}, \quad \text { for } j<m \\
y_{m}^{p} & >z_{m}^{p}
\end{aligned}
$$

Please refer to the Appendix A for an illustrative example of the preference relation and the lexicographic maximin solution.

Definition 1 The lexicographic maximin objective vector $\boldsymbol{z}^{\text {lex }}$ is the largest with respect to $\succ$, i.e. $\nexists \boldsymbol{z} \in \mathcal{Z}$ such that $\boldsymbol{z} \succ \boldsymbol{z}^{\text {lex }}$.

Basic Approach: From Def. 1, a general approach to find the lexicographic maximin solution is to first improve the utility of the worst-off individuals until there is no feasible way to go further. Then keeping these allocations, repeat the procedure under the remaining problem until all the individuals get their final allocations.

Note that the lexicographic maximin criterion defined above differs from simple lexicographic ordering commonly used to sort out multiple objective problems. While the latter requires a strict precendence order according to the importance previously attributed to each utility function, in the first criterion there is no such order, all utility functions have the same importance being maximised altogether, they are limited solely by intrinsic problem constraints.

The advantage of characterising the lexicographic maximin solution from the objective set is that it is usually of lower dimension than the domain set, which may simplify the analysis. From Def. 1 some important $\boldsymbol{z}^{l e x}$ properties are readily available:

(i) $\boldsymbol{z}^{\text {lex }}$ exists if $\mathcal{Z} \neq \varnothing$

(ii) $\boldsymbol{z}^{\text {lex }}$ is unique in the objective set

(iii) $\pi\left(\boldsymbol{z}^{l e x}\right)=\boldsymbol{z}^{l e x}$

(iv) $\boldsymbol{z}^{\text {lex }}$ is Pareto Efficient by definition.

\subsection{Algorithmic Determination}

Since the closed-form expression in (5) is not adequate for optimisation we will not take it into further regard, the approach based on Def. 1 will be used instead to allow an

\footnotetext{
${ }^{1}$ For $\boldsymbol{z}=(0.7,0.9,0.3,0.1)$ then $\pi(\boldsymbol{z})=(0.1,0.3,0.7,0.9)$ and $z_{1}^{p}=0.1, z_{2}^{p}=0.3, z_{3}^{p}=0.7, z_{4}^{p}=0.9$
} 
algorithmic determination of the lexicographic maximin solution. The idea is to build up the $\boldsymbol{z}^{\text {lex }}$ vector from the smallest to the largest components following the order in which they are produced, in other words, first improve the utility of the worst-off individuals until there is no feasible way to go further. This can be achieved from the following problem:

$$
\max _{\boldsymbol{x} \in \mathcal{X}}\{h \mid u(\boldsymbol{x}, j) \geq h ; \forall j \in \mathcal{N}\}
$$

If all the inequality constraints are binding in the solution of (10), there is no feasible way to increase the utility of a given flow without decreasing the utility of another flow, hence by Def. 1 we have the lexicographic maximin allocation. In case that not all constraints are binding, let $\mathcal{S} \subset \mathcal{N}$ be the set of binding constraints. For all commodity flows $j \in \mathcal{S}$ there is no feasible way to increase their utilities altogether, they are the worst-off commodity flows in the lexicographic maximin solution (the smallest components of the $\boldsymbol{z}^{\text {lex }}$ vector). For the remaining commodity flows $j \in \mathcal{N} \backslash \mathcal{S}$, the problem must continue in the next step at higher utilities (lexicographic order).

Assume $h(1)$ as the solution of (10), i.e. the maximum value obtained for $h$ over all feasible allocations $\boldsymbol{x} \in \mathcal{X}$. The next step is to check which commodity flows $j \in \mathcal{N}$ are not yet blocked or analogously which $\boldsymbol{z}^{\text {lex }}$ components can be increased beyond $h(1)$. If there is no component that can be made greater than $h(1)$ without making another one smaller than $h(1)$, all $\boldsymbol{z}^{\text {lex }}$ components are equal to $h(1)$.

Let $\boldsymbol{x}^{*}$ be an allocation that solves $(10)$, if $u\left(\boldsymbol{x}^{*}, j\right)>h(1)$, flow $j$ is not yet blocked. In fact, a flow $i \in \mathcal{N}$ is blocked if,

$$
\max _{\boldsymbol{x} \in \mathcal{X}}\{u(\boldsymbol{x}, i) \mid u(\boldsymbol{x}, j) \geq h(1) ; \forall j \in \mathcal{N} ; j \neq i\}=h(1)
$$

Let $\mathcal{B}(1) \subseteq \mathcal{N}$ represents the set of blocked commodity flows satisfying (11), if $\mathcal{B}(1)=$ $\mathcal{N}$ we stop, otherwise there are still some unblocked flows and a new problem has to be solved at a second stage without wasting the previous solution,

$$
h(2)=\max _{\boldsymbol{x} \in \mathcal{X}}\left\{h \mid u(\boldsymbol{x}, j) \geq h ; u\left(\boldsymbol{x}, i_{1}\right)=h(1) ; \forall j \in \mathcal{N} \backslash \mathcal{B}(1) ; \forall i_{1} \in \mathcal{B}(1)\right\}
$$

The procedure is repeated until all commodity flows get blocked, at this point we have the lexicographic maximin solution by construction.

Generally, at stage $k$,

$$
\begin{gathered}
h(k)=\max _{\boldsymbol{x} \in \mathcal{X}}\left\{h \mid u(\boldsymbol{x}, j) \geq h ; u\left(\boldsymbol{x}, i_{1}\right)=h(1), \ldots, u\left(\boldsymbol{x}, i_{k-1}\right)=h(k-1)\right. \\
\left.\forall i_{1} \in \mathcal{B}(1), \ldots, \forall i_{k-1} \in \mathcal{B}(k-1) ; \forall j \in \mathcal{N} \backslash \mathcal{B}\right\}
\end{gathered}
$$


where $\mathcal{B}=\bigcup_{m=1}^{k-1} \mathcal{B}(m)$. The set $\mathcal{B}(k)$ contains all commodity flows $i \in \mathcal{N} \backslash \mathcal{B}$, such that:

$$
\begin{gathered}
\max _{\boldsymbol{x} \in \mathcal{X}}\left\{u(\boldsymbol{x}, i) \mid u(\boldsymbol{x}, j) \geq h(k) ; u\left(\boldsymbol{x}, i_{1}\right)=h(1), \ldots, u\left(\boldsymbol{x}, i_{k-1}\right)=h(k-1)\right. \\
\left.\forall j \in \mathcal{N} \backslash \mathcal{B} ; j \neq i ; \forall i_{1} \in \mathcal{B}(1), \ldots, \forall i_{k-1} \in \mathcal{B}(k-1) ;\right\}=h(k)
\end{gathered}
$$

The procedure is summarized next:

\section{PROCEDURE-1}

1. $k \leftarrow 1$

2. compute $h(k)$ from $(13)$

3. compute $\mathcal{B}(k)$ using (14), and update $\mathcal{B}$

4. if $\mathcal{N}=\mathcal{B}$

return $\boldsymbol{x}^{\text {lex }}$ from last solution of (13)

else

$k \leftarrow k+1$

goto 2 .

end

From the procedure just described and from the monotonicity of the utility functions, it can be directly verified that:

$$
h(1)<h(2)<\ldots<h(k-1)<h(k)<h(k+1) \ldots
$$

In other words, $h(k)$ defines an increasing sequence on the index $k$. Such sequence reflects the order embedded in the lexicographic maximin criterion and serves as a framework to build up algorithmic solutions. Each $h(k)$ establishes the utility levels in which one or more commodity flows get blocked since at this situation there is no feasible way to increase their utilities without decreasing the utilities of other worse-off commodity flows.

In the worst case scenario Procedure- 1 needs $N$ iterations to complete since at each stage at least one flow gets blocked. Although, Procedure-1 constitutes an alternative to overcome the difficulty in solving problem (5), it requires the solution of expensive nonlinear optimisation problems in (13) and (14). In practical network scenarios the number of utility functions, variables and constraints are usually large which makes the solution of such non-linear problems a serious burden.

In fact, the purpose of Procedure- 1 is to show that the lexicographic maximin solution can always be obtained for the problems of interest, and also to lay down the basic 
framework from where more practical implementations can be deployed. The next section investigates such implementations and proposes a more convenient algorithm to determine the lexicographic maximin solution.

\section{The Maximum Concurrent Multicommodity Flow Algorithm}

The general idea of this approach is to propose a solution to the sequence of non-linear subproblems described in Procedure-1 through the use of a sequence of all linear subproblems.

Multicommodity Flow Problems (MFP) have been the subject of great interest in the Operations Research area given their applicability to model and solve a wide range of practical problems: routing of multiple commodities, warehousing of seasonal products, multivehicle tanker scheduling (Ahuja et al. 1993). Recently some specialized interiorpoint algorithms have been reported to solve large problem instances of up to 2.5 million variables and 260,000 constraints (Castro, 2000a, 2000b). Hence our aim is to build up an algorithm based on a series of MFP subproblems instead of the non-linear subproblems in (13) and (14), with the view of employing those specialized methods to overcome potential scalability difficulties. Moreover, since MFP are indeed linear programming problems general linear programming code may be used as well.

We start applying a particular type of MFP, the maximum concurrent multicommodity flow problem - MCMF (Shahrokhi and Matula, 1990), to serve as a building block for our proposed algorithm:

$$
\lambda^{*}(\boldsymbol{d})=\max \left\{\lambda \mid \sum_{\boldsymbol{x} \in \mathcal{X}}^{\left|P_{j}\right|} x_{j k}=\lambda d_{j} ; \forall j \in \mathcal{N}\right\}
$$

where $\boldsymbol{d}$ represents the vector of commodity demands, $\boldsymbol{d}:\left(d_{1}, d_{2}, \ldots, d_{N}\right)$, and $\lambda^{*}(\boldsymbol{d})$ the solution of the above problem for a given $\boldsymbol{d}$.

The idea is to apply (16) to solve (10) and more generally (13). The rationale behind this approach is the following. First, observe that both problems (10) and (16) have same objective functions and domain sets $\mathcal{X}$. Then, starting from a feasible point, say $\boldsymbol{x}=\mathbf{0}$, objective function maximization implies in commodity flows inflation until capacity constraints bind and block further inflations. So the objective function works as an inflation parameter. In problem (10) the parameter $h$ inflates flows according to their utility functions, while in problem (16) parameter $\lambda$ inflates flows directly according to $d_{j}$. It will be 
seen next that by adjusting the values of $d_{j}$ and solving (10) iteractively it is possible to arrive at a solution for (16).

Let $\boldsymbol{x}^{e}$ be a feasible point $\left(\boldsymbol{x}^{e} \in \mathcal{X}\right)$ such that $u\left(\boldsymbol{x}^{e}, j\right)=h, \forall j \in \mathcal{N}$, or in other words, $\boldsymbol{x}^{e}$ leads to an objective vector $\boldsymbol{z}^{e}$ with equal coordinates: $\boldsymbol{z}^{e}=(h, h, \ldots, h) ; h>0$ (refer to Appendix B for a proof on the existence of this point). For a vector $\boldsymbol{d}$ with components given by $d_{j}=\sum_{k=1}^{\left|P_{j}\right|} x_{j k}^{e}$ (or alternatively $d_{j}=u^{-1}(h, j)$, where the inverse $u^{-1}(h, j)$ returns the amount of overall flow that gives to commodity $j$ an utility $h$ ) we have at the solution,

$$
\lambda^{*}(\boldsymbol{d})=\frac{\sum_{k=1}^{\left|P_{j}\right|} x_{j k}^{*}}{\sum_{k=1}^{\left|P_{j}\right|} x_{j k}^{e}} \quad \forall j \in \mathcal{N}
$$

Since $\boldsymbol{x}^{e} \in \mathcal{X}, \lambda^{*}(\boldsymbol{d})<1$ contradicts the optimality of problem $(16) \Rightarrow \lambda^{*}(\boldsymbol{d}) \geq 1$.

In case $\lambda^{*}(\boldsymbol{d})=1$, from (17) we have that $u\left(\boldsymbol{x}^{*}, j\right)=h, \forall j \in \mathcal{N}$, and since utilities are monotone, there is no feasible way to increase the utilities altogether above $h \Rightarrow \boldsymbol{x}^{*}$ also solves (10).

For the other case, $\lambda^{*}(\boldsymbol{d})>1$, let

$$
\begin{aligned}
\bar{u} & =\min _{j \in \mathcal{N}}\left\{u\left(\boldsymbol{x}^{*}, j\right)\right\} \\
\alpha_{j} & =\frac{u^{-1}(\bar{u}, j)}{\sum_{k=1}^{\left|P_{j}\right|} x_{j k}^{*}}
\end{aligned}
$$

From the monotonicity of the utility functions we have $\bar{u}>h$ and $0<\alpha_{j} \leq 1$. Let $\bar{x}_{j k}=\alpha_{j} x_{j k}^{*} \Rightarrow \overline{\boldsymbol{x}} \in \mathcal{X}$ since $\boldsymbol{x}^{*} \in \mathcal{X}$ and all path variables were multiplied by a number between 0 and 1 . We have also,

$$
\alpha_{j} \sum_{k=1}^{\left|P_{j}\right|} x_{j k}^{*}=\sum_{k=1}^{\left|P_{j}\right|} \alpha_{j} x_{j k}^{*}=\sum_{k=1}^{\left|P_{j}\right|} \bar{x}_{j k}=u^{-1}(\bar{u}, j) \Rightarrow u(\overline{\boldsymbol{x}}, j)=\bar{u} \quad \forall j \in \mathcal{N}
$$

Since $\bar{u}>h$ and $u\left(\boldsymbol{x}^{*}, j\right) \geq \bar{u}$, the following relations are valid for all $j \in \mathcal{N}$ :

$$
\begin{gathered}
u\left(\boldsymbol{x}^{e}, j\right)<u(\overline{\boldsymbol{x}}, j) \leq u\left(\boldsymbol{x}^{*}, j\right) \\
\sum_{k=1}^{\left|P_{j}\right|} x_{j k}^{e}<\sum_{k=1}^{\left|P_{j}\right|} \alpha_{j} x_{j k}^{*} \leq \sum_{k=1}^{\left|P_{j}\right|} x_{j k}^{*} \\
d_{j}<\bar{d}_{j} \leq d_{j}^{*}
\end{gathered}
$$

Thus, from $\boldsymbol{x}^{e}$ we can move to a point $\overline{\boldsymbol{x}}$ of increased demands $\left(\bar{d}_{j}>d_{j}\right)$ and identical 
utilities that are strictly higher $(\bar{u}>h)$ than before. That is:

$$
\lambda^{*}(\boldsymbol{d})\left\{\begin{array}{l}
=1, \quad \text { the solution of }(16) \text { also solves }(10) \\
>1, \quad \text { demands and utilities can be increased to } \overline{\boldsymbol{d}} \text { and } \bar{u}
\end{array}\right.
$$

If the demands are updated in problem (16) to the new values of $\bar{d}_{j}$ and the procedure is repeated all over again, we generate an increasing sequence of demands $\bar{d}_{j}$ and utilities $\bar{u}$ until we eventually arrive to the condition that $\lambda^{*}(\boldsymbol{d})=1$, since demands cannot be increased indefinitely. At this point, $\boldsymbol{x}^{*}$ solves problem (10) with $h(1)$ given by the last returned $\bar{u}$. This constitutes the main idea behind our proposed MCMF approach and it will be seen next how this property can be used to construct an algorithm to find the optimal solution $\boldsymbol{x}^{\text {lex }}$.

Before that, let us take the more general problem (13). The non-linear equality constraints in (13) are in the form of $u(\boldsymbol{x}, j)=h$ which are equivalent to $\sum_{k=1}^{\left|P_{j}\right|} x_{j k}=u^{-1}(h, j)$. Thus, we can rewrite the equation as,

$$
\begin{gathered}
h(k)=\max _{\boldsymbol{x} \in \mathcal{X}}\left\{h \mid u(\boldsymbol{x}, j) \geq h ; \sum_{m=1}^{\left|P_{i_{1}}\right|} x_{i_{1}, m}=u^{-1}\left(h(1), i_{1}\right), \ldots, \sum_{m=1}^{\left|P_{i_{k-1}}\right|} x_{i_{k-1}, m}=\right. \\
\left.u^{-1}\left(h(k-1), i_{k-1}\right) ; \quad \forall i_{1} \in \mathcal{B}(1), \ldots, \forall i_{k-1} \in \mathcal{B}(k-1) ; \forall j \in \mathcal{N} \backslash \mathcal{B}\right\}
\end{gathered}
$$

Note that all equality constraints have the same form and can be written as $\sum_{m=1}^{\left|P_{i}\right|} x_{i m}=d_{i}$, where for each flow $i$ the value of $d_{i}$ is fixed and corresponds to the associated inverse utility. Therefore, the problem can be expressed in terms of linear equality constraints only,

$$
h(k)=\max _{\boldsymbol{x} \in \mathcal{X}}\left\{h \mid u(\boldsymbol{x}, j) \geq h ; \sum_{m=1}^{\left|P_{i_{1}}\right|} x_{i_{1}, m}=d_{i} ; \forall i \in \mathcal{B} ; \forall j \in \mathcal{N} \backslash \mathcal{B}\right\}
$$

where $\mathcal{B}=\bigcup_{m=1}^{k-1} \mathcal{B}(m)$.

If we know beforehand the set $\mathcal{B}$ and the values of $d_{i}$, we can apply the same recursive procedure explained in this section over the extended MCMF problem,

$$
\lambda^{*}(\boldsymbol{d})=\max _{\boldsymbol{x} \in \mathcal{X}}\left\{\lambda \mid \sum_{k=1}^{\left|P_{j}\right|} x_{j k}=\lambda d_{j} ; \forall j \in \mathcal{N} \backslash \mathcal{B} ; \sum_{k=1}^{\left|P_{i}\right|} x_{i k}=d_{i} ; \forall i \in \mathcal{B}\right\}
$$

in order to solve (25) or equivalently solve (13). Similarly, $h(k)$ will be given by the last computed $\bar{u}$.

The only remaining step necessary for the construction of an algorithm that is equiv- 
alent to Procedure -1 regards to the determination of blocked commodity flows and their corresponding values of $d_{i}$. Algorithm-2 presented next includes such step and provides a full replacement for Procedure-1 with the advantage of not relying on any non-linear optimisation subproblem.

\section{ALGORITHM-2}

0. $\mathcal{B} \leftarrow \varnothing$ "set of blocked flows"

$$
\text { for each } j \in \mathcal{N}: d_{j} \leftarrow \epsilon \text { end }
$$

1. solve problem $(26)$,

$$
\begin{aligned}
& \lambda^{*}(\boldsymbol{d})=\max _{\boldsymbol{x} \in \mathcal{X}}\left\{\lambda \mid \sum_{k=1}^{\left|P_{j}\right|} x_{j k}=\lambda d_{j} ; \forall j \in \mathcal{N} \backslash \mathcal{B} ; \sum_{k=1}^{\left|P_{i}\right|} x_{i k}=d_{i} ; \forall i \in \mathcal{B}\right\} \\
& \mathcal{X}=\left\{\sum_{j \in \mathcal{N}} \sum_{k=1}^{\left|P_{j}\right|} \delta_{l}\left(p_{j k}\right) x_{j k} \leq C_{l} ; \quad x_{j k} \geq 0 ; \quad l \in \mathcal{L}\right\}
\end{aligned}
$$

2. if $\lambda^{*}>1$

$$
\begin{aligned}
& \bar{u} \leftarrow \min _{j \in \mathcal{N} \backslash \mathcal{B}}\left\{u\left(\lambda^{*} d_{j}, j\right)\right\} \\
& d_{j} \leftarrow u^{-1}(\bar{u}, j) \quad \forall j \in \mathcal{N} \backslash \mathcal{B} \\
& \text { goto Step } \mathbf{1 .}
\end{aligned}
$$

end

3. $\mathcal{B}_{L} \leftarrow \varnothing$ "set of saturated links"

for each $l \in \mathcal{L}$

if constraint active: $\mathcal{B}_{L} \leftarrow \mathcal{B}_{L} \cup\{l\}$ end

end

for each $j \in \mathcal{N} \backslash \mathcal{B}$

find $p \in P_{j}$ in $\mathcal{G}=\left(\mathcal{V}, \mathcal{L} \backslash \mathcal{B}_{L}\right)$

if no path: $\mathcal{B} \leftarrow \mathcal{B} \cup\{j\}$ end

end

4. if $\mathcal{B}=\mathcal{N}:$ END

else: $\quad$ goto Step 1.

The box diagram in Fig.1 shows the step-by-step functional correspondence between Procedure -1 and Algorithm-2. The non-linear problem in step (2) of Procedure-1 is solved in Algorithm-2 through steps (1)-(2) loop. Also, the determination of set $\mathcal{B}$ follows a different procedure in Algorithm-2.

Fig.1: Functional correspondence between Procedure-1 and Algorithm-2. 
Step (0) in Algorithm-2 creates the set of blocked commodity flows $\mathcal{B}$ initially empty. Demands are also initiated to a small value $\epsilon$ so that they are feasible in any network $\mathcal{G}=(\mathcal{V}, \mathcal{L})$ of interest.

Step (1) involves the solution of the extended MCMF sub-problem, which in the first iteration is identical to (10). In fact, this step together with Step (2) implements the recursive approach explained from (16) to (26). The parameter $\lambda^{*}$ indicates how much the unblocked commodity flows can be inflated without violating any capacity constraints. Assuming $\lambda^{*}>1$, Step (2) is executed to obtain the minimum utility among the unblocked commodity flows and the corresponding value of demands for that utility. As long as $\lambda^{*}>1$, steps (1) and (2) are repeated in turns and the utility of the worst-off individual $(\bar{u})$ increases at each iteration. Since utility functions are monotone and demands cannot be increased indefinitely due to capacity constraints, $\lambda^{*}$ eventually reaches 1 . At this stage it is not possible to increase the minimum utility in the system and the current domain point solves problem (10).

Step (3) verifies which commodity flows are blocked and updates the set $\mathcal{B}$ accordingly. If all commodity flows are blocked, $\mathcal{N}=\mathcal{B}$ in Step (4), the algorithm terminates otherwise it continues with another expansion in Step (1). In the first loop of Step (3) the set of saturated links is built such that $l \in \mathcal{B}_{L}$ if

$$
\sum_{j \in \mathcal{N}} \sum_{k=1}^{\left|P_{j}\right|} \delta_{l}\left(p_{j k}\right) x_{j k}^{*}=C_{l}
$$

The second loop checks if a flow $j$ is blocked by verifying if there still exists paths in $P_{j}$ with available bandwidth. The whole step serves as an alternative of lower complexity for the determination of the blocking sets in (14).

Note that blocked commodity flows $(i \in \mathcal{B})$ do not change their utilities anymore since in the next call to Step (2), $\sum_{k=1}^{\left|P_{i}\right|} x_{i k}$ will be fixed. Note also that for these commodity flows $d_{i}$ will be given by the last update in Step (2), which for the first algorithm iteration is $u^{-1}(h(1), i)$.

Commodity flows that are unblocked continue to be inflated and are allowed to experience higher utilities. A new algorithm iteration start again in Step (1) and whenever $\lambda^{*}$ reaches 1 in Step (2), a solution for problem (24) is returned with $h(k)$ given by the last computed $\bar{u}$. In fact, Algorithm-2 mimics Procedure-1 without solving complex non-linear programming problems. 


\subsection{Complexity}

The algorithm complexity in terms of the maximum number of operations required to complete each step is discussed here, we use the following notation: $\boldsymbol{l}$ represents the number of links in the topology, $\boldsymbol{v}$ the number of nodes, $\boldsymbol{m}$ the number of commodity flows in the system, and $\boldsymbol{n}$ the overall number of path variables.

Since step (0) is only executed in the first iteration and is much simpler than the other steps it is not considered. Step (1) involves the solution of a MCMF problem, which according to (Stein, 1992) can be solved in $O\left(\epsilon^{-2} \boldsymbol{m}^{2} \boldsymbol{l} \boldsymbol{v} \log \boldsymbol{m} \log ^{3} \boldsymbol{v}\right)$, where $\epsilon$ is the precision required. ${ }^{2}$ Steps (2) and (3) complexities are respectively $O(\boldsymbol{m})$ and $O\left(\boldsymbol{l}+\boldsymbol{m} \boldsymbol{v}^{2}\right)$. The latter is due to a maximum of $\boldsymbol{l}$ operations to complete the first loop, and a maximum of $\boldsymbol{m}$ shortest-path computations (the complexity of Dijkstra's algorithm is $O\left(\boldsymbol{v}^{2}\right)$ ) to complete the second loop.Hence, the complexity of all steps is $O\left(\epsilon^{-2} \boldsymbol{m}^{2} \boldsymbol{l} \boldsymbol{v} \log \boldsymbol{m} \log ^{3} \boldsymbol{v}+\right.$ $\boldsymbol{m} \boldsymbol{v}^{2}$ ). What can be seen is that in most cases step (1) dominates the other steps, and the complexity of an algorithm iteration can be considered as the complexity of this step itself. Roughly speaking, and when $m$ reaches a considerable large magnitude Algorithm2 iteration is as difficult as a linear MCMF problem.

\subsection{Requirements and Limitations}

Algorithm-2 main requirement is the use of a linear programming solver or a specialized MCMF solver to carry out step (1). Although such solvers are widely available on optimisation packages, mathematics softwares and even distributed freely as standalone $\operatorname{codes}^{3}$, algorithm implementation performance may vary according to the solver package used. For very large problems it seems that specialized MCMF codes perform better than standard linear programming codes (Castro, 2000a, 2000b) . However, the constant improvements and new releases of some high-performance linear programming packages ${ }^{4}$ may make the specialized codes not necessary.

The other requirement of Algorithm-2 is to employ a shortest-path procedure in step (3) in order to check for blocked commodity flows. Since most of solvers return a list of active constraints at the solution point, this type of test can be directly executed. If we take out from the topology all the links with active constraints, the remaining topology will contain only links with spare resources. Therefore, a flow is blocked if there is no available path on the remaining topology. Alternatively, an all-pairs shortest path procedure returns paths only for unblocked commodity flows and could also be used.

\footnotetext{
${ }^{2}$ Stein's algorithm returns a solution within a factor of $(1-\epsilon)$ from the exact optimal.

${ }^{3}$ The non-commercial linear programming code, lp solve, was written in ANSI C and is publicly available

${ }^{4}$ e.g. CPLEX 8.0, http://www.cplex.com
} 


\section{Numerical Results}

In this section we compare the solutions returned by our proposal with the results from the two most well-known approaches in the networking area: max-min (Bertsekas and Gallager, 1992) and proportional fairness (Kelly et al. 1998), both extended to the multipath environment. We are interested in assessing the quality of the solution in terms of minimum and average utility (utility of the system), and the gains that may be achieved by using our approach.

Three network topologies were selected for the experiments: JANET - Fig.2(a), GEANT - Fig.2(b), and 47-NODE - Fig.2(c). The topologies differ in number of nodes, links, symmetry, and level of connectivity. We assume that a capacity of $100 \mathrm{Mbps}$ was reserved for the experiments in all network links and should be adequately allocated to the different applications in the system.

Fig.2(a): JANET topology, Fig.2(b): GEANT topology, Fig.2(c): 47NODE topology

Applications may generate traffic from any network node (origin) to any other node (destination) and have an associated utility function on the form of the logarithmic expressions presented in (Richards et al. 1998):

$$
\begin{gathered}
u(x, j)=a_{j} \cdot \ln \left(b_{j} x+c_{j}\right), \quad u(I, j)=1, \quad u(M, j)=0 \\
a_{j}=\frac{1}{k_{j}-10}, \quad b_{j}=\frac{e^{\frac{1}{a}}-1}{I-M}, \quad c_{j}=\frac{I-M e^{\frac{1}{a}}}{I-M}
\end{gathered}
$$

where $I$ represents the ideal allocation and $M$ the minimum requirement. By changing the parameter $k$ different functions can be obtained: strictly concave for $k>10$, linear for $k \rightarrow 10$, and convex for $k<10$.

Figure 3 shows a plot of such expressions for $M=10 \mathrm{Kpbs}$ (minimum requirement, utility $=0$ ), $I=100 \mathrm{Kbps}$ (ideal, utility $=1$ ), and $k=\{5,6, \ldots, 15\}$ (concavity parameter).

Fig.3: Logarithm-based utility functions for 11 different values of $k$.

The bandwidth allocation solution under the lexicographic maximin criterion was computed using Algorithm-2 and compared here with max-min and proportional fairness solutions extended to the multi-path environment.

We evaluate the quality of each solution returned in terms of minimum and average 
utility in the system:

$$
\begin{aligned}
\bar{u} & =\min _{j \in \mathcal{N}}\{u(\boldsymbol{x}, j)\} \\
U & =\frac{1}{|\mathcal{N}|} \sum_{j \in \mathcal{N}} u(\boldsymbol{x}, j)
\end{aligned}
$$

The minimum utility, $\bar{u}$, gives a lower bound for individual application performance, while the average utility, $U$, reflects the performance of the system as a whole. The solution of higher minimum utility is preferred under a Rawlsian perspective, while a higher average utility is more desirable from an utilitarian viewpoint.

The system was tested with 10,000 individual applications whose utility function were described by the logarithmic curves in Fig.3. A total of 20 scenarios were considered in the experiment, where for each scenario a different and random assignment of commodity flows to OD pairs and utility functions took place.

For each one of the 20 scenarios we computed the minimum and average utility under max-min fairness $\left(\bar{u}_{m m f}, U_{m m f}\right)$, proportional fairness $\left(\bar{u}_{p p f}, U_{p p f}\right)$, and the lexicographic maximin criterion $\left(\bar{u}_{\text {lex }}, U_{\text {lex }}\right)$. We then computed the respective ratios: $\bar{u}_{\text {lex }} / \bar{u}_{m m f}$, $\bar{u}_{l e x} / \bar{u}_{p p f}, U_{l e x} / U_{m m f}, U_{l e x} / U_{p p f}$ and summarized the results obtained in the tables below.

\begin{tabular}{|ccc|ccc|}
\hline \multicolumn{6}{|c|}{ JANET } \\
\hline \multicolumn{4}{|c|}{ Lexicographic Maximin $/$ max-min } \\
\hline $\bar{u}_{\text {lex }} / \bar{u}_{\text {mmf }}$ & \multicolumn{4}{c|}{$U_{\text {lex }} / U_{\text {mmf }}$} \\
lowest & mean & highest & lowest & mean & highest \\
1.59 & 2.69 & 4.13 & 0.97 & 1.02 & 1.11 \\
\hline
\end{tabular}

\begin{tabular}{|ccc|ccc|}
\hline \multicolumn{6}{|c|}{ JANET } \\
\hline \multicolumn{3}{|c|}{ Lexicographic Maximin / Proportional } \\
\hline \multicolumn{3}{|c|}{$\bar{u}_{\text {lex }} / \bar{u}_{p p f}$} & \multicolumn{3}{c}{$U_{\text {lex }} / U_{\text {ppf }}$} \\
lowest & mean & highest & lowest & mean & highest \\
1.62 & 3.33 & 6.34 & 0.90 & 1.00 & 1.08 \\
\hline
\end{tabular}

Table 1: Summary of the solutions obtained (max-min, proportional, lexicographic maximin) for each of the 20 scenarios generated for the JANET topology. 


\begin{tabular}{|ccc|ccc|}
\hline \multicolumn{6}{|c|}{ GEANT } \\
\hline \multicolumn{4}{|c|}{ Lexicographic Maximin / max-min } \\
\hline lowest & $\bar{u}_{\text {lex }} / \bar{u}_{\text {mmf }}$ & \multicolumn{4}{c|}{$U_{\text {lex }} / U_{\text {mm }}$} \\
1.37 & 2.35 & 3.56 & 0.85 & 0.99 & 1.15 \\
\hline
\end{tabular}

\begin{tabular}{|ccc|ccc|}
\hline \multicolumn{6}{|c|}{ GEANT } \\
\hline \multicolumn{3}{|c|}{ Lexicographic Maximin / Proportional } \\
\hline \multicolumn{3}{|c|}{$\bar{u}_{\text {lex }} / \bar{u}_{p p f}$} & \multicolumn{4}{c|}{$U_{\text {lex }} / U_{\text {ppf }}$} \\
lowest & mean & highest & lowest & mean & highest \\
1.38 & 2.50 & 4.42 & 0.81 & 0.97 & 1.09 \\
\hline
\end{tabular}

Table 2: Summary of the solutions obtained (max-min, proportional, lexicographic maximin) for each of the 20 scenarios generated for the GEANT topology.

\begin{tabular}{|ccc|ccc|}
\hline \multicolumn{5}{|c|}{ 47-NODE } \\
\hline \multicolumn{4}{|c|}{ Lexicographic Maximin / } & \multicolumn{3}{c|}{$U_{\text {lex }} / U_{\text {mm }}$} \\
$\bar{u}_{\text {lex }} / \bar{u}_{\text {mmf }}$ & \multicolumn{3}{c|}{} \\
lowest & mean & highest & lowest & mean & highest \\
1.22 & 2.47 & 4.28 & 0.92 & 1.07 & 1.34 \\
\hline
\end{tabular}

\begin{tabular}{|ccc|ccc|}
\hline \multicolumn{6}{|c|}{ 47-NODE } \\
\hline \multicolumn{4}{|c|}{ Lexicographic Maximin / Proportional } \\
\hline \multicolumn{3}{|c|}{$\bar{u}_{\text {lex }} / \bar{u}_{p p f}$} & \multicolumn{4}{c|}{$U_{\text {lex }} / U_{p p f}$} \\
lowest & mean & highest & lowest & mean & highest \\
1.47 & 3.02 & 4.8 & 0.85 & 1.03 & 1.27 \\
\hline
\end{tabular}

Table 3: Summary of the solutions obtained (max-min, proportional, lexicographic maximin) for each of the 20 scenarios generated for the 47-NODE topology. 
On each table the first and forth columns correspond to the lowest ratio observed over the 20 scenarios, the second and fifth columns correspond to the mean ratios over all scenarios, and finally the third and sixth columns correspond to the highest ratio observe over the 20 scenarios for each network topology.

From all the results presented it can be seen a much better performance of the system in terms of minimum utility for the lexicographic maximin criterion since all the ratios were above one - higher minimum utility under the lexicographic maximin solution than under max-min or proportional fairness. In this case, the overall lowest gain was $22 \%$ observed for the 47-NODE topology as indicated in Table 3, in other words, the worst performance under the lexicographic maximin criterion was still $22 \%$ better than the other schemes.

In terms of average utility in the system, there was no significant difference between lexicographic maximin solutions and max-min or proportional solutions. In all tables, the fifth column indicates that mean values were also very close to one, which does not give a clear advantage to any of the schemes.

Regarding computational times, the lexicographic maximin solution demanded more processing time than the other two approaches, as expected. For the largest problems (47NODE topology) it may take up to 30 minutes to return a lexicographic maximin solution running over an Intel Pentium IV 2.66GHz, 512 Mbytes RAM. Mean running times were about 2 minutes (max-min), 3 minutes (proportional) and 6 minutes (lexicographic).

\section{Discussion}

This section discusses some limitations, considerations and practical issues related to the proposed approach.

In this paper we considered continuous and monotone utility functions as functions of network throughput assigned to commodity flows. This model covers traditional internet applications (file transfers, e-mail, web), which are reported to have strictly concave utility functions (Sheker, 1995). The model also applies to some multimedia applications such as audio and video with "internet quality" that are able to change their throughput and encoding schemes to adapt to network congestion (Diot, 1995). These applications are also known as soft-QoS (rate and delay adaptive) and can be modeled by S-shaped utility curves (Shenker, 1995). Our approach does not apply to strict-QoS applications which may be modeled by discontinuous functions or by other network metrics as delay, loss, etc.

The solution presented in this paper gives a theoretical optimal point in terms of fairness and efficiency. In practice it will be difficult to maintain the network operating 
exactly at this point, however the point serves as a goal to be pursued by network protocols and mechanisms. One way to enforce such allocation is to employ QoS mechanisms and architectures, such as MPLS, DiffServ, WFQ, RED, etc. For instance, individual commodity flows which are identical (same utility functions) could be aggregated and the overall amount of resources for the aggregated flow be allocated on the path using the MPLS protocol. This approach guarantees allocations at the aggregated level which are less dynamic and more scalable. Inside the aggregates, it is important to avoid that a single individual flow consumes all the resources and leave all other commodity flows starved. Buffer management schemes could be employed along the path to perform selective dropping of packets and correct this situation, e.g. RED dropper. Note that this scheme is compatible with commodity flows that use congestion control algorithms such as the TCP protocol.

The use of MPLS and QoS mechanisms gives also support to applications that require priority (strict-QoS). At the expenses of a more complex implementation procedure, separate allocations could be set for these applications so that the network preserves our fair and efficient solution and through prioritization attend strict-QoS traffic in other partition.

There are other types of network traffic not directly attended by our framework, for instance peer-to-peer network traffic and bursty traffic. One way to map this traffic to our framework is to determine its effective bandwidth, and then by using this parameter the allocations could be done as studied in this paper. An interesting reference to this topic is (Kelly et al. , 1996). An extension of this paper and hence a topic of further research would be to consider other types of traffic with e.g. self-similarity and/or long range dependence characteristics.

Finally, we briefly comment about fairness and pricing. The importance of fairness to the allocation of network resources has been tackled by several works. In (Denda et al., 2000) and (Bonald and Massoulie, 2001) thorough discussions are presented about why fairness should be considered in the network. The last work mathematically proves that fairness stabilizes the network and so it is a performance parameter for network operators. In relation to pricing, flat rate is by far the most widely used and customer preffered pricing scheme. We believe that this scheme would work well with our proposal. Another scheme that may also apply is to charge commodity flows according to their profile (utility function), in this way there will be incentives for the user to choose the most adequate profile for his traffic. 


\section{Conclusion}

In this paper the bandwidth allocation problem was studied under a multi-application network environment. Each application was represented by a monotone utility function, which depends on the amount of allotted bandwidth. We formulated the problem as a constrained multiple objective optimisation problem (CMOP) and employed the lexicographic maximin criterion to resolve conflicts and return a solution that satisfied fairness and efficiency properties.

A new algorithm was proposed to overcome the difficulty on returning lexicographic maximin allocation solutions. The algorithm was based on maximum concurrent multicommodity flow approach and made use of linear multicommodity flow subproblems to tackle the nonlinearity introduced by the utility functions. Such solution may benefit from current and future specialized multicommodity flow or linear programming solvers to solve large instances of the problem.

Tests were carried out using a variety of network topologies, utility functions, and flow assignments to verify the gains produced by our approach when compared to other relevant proposals. From the results presented, the lexicographic maximin criterion appeared to be a promising way to sort out bandwidth allocation over multi-application environments specially if fairness is to be considered.

However, while the lexicographic maximin criterion guarantees desirable features for the allocation of network resources such as fairness and efficiency, it requires complex optimisation procedures to find a solution. A possible future work consists of investigating approximated versions for the lexicographic maximin criterion, which would be more tractable and could be expressed by a closed-form expression.

\section{Appendix A. Lexicographic maximin solution example}

Consider a system with five individuals and four feasible allocations: $\mathcal{N}=\left\{i_{1}, i_{2}, i_{3}, i_{4}, i_{5}\right\}$ and $\mathcal{X}=\{a, b, c, d\}$. The utility achieved by each individual for the feasible allocations, and the corresponding objective vectors are given by Fig.4.

\section{Fig.4: Lexicographic maximin example}

From the above table, we have $z_{d} \succ z_{a}$ since the minimum utility at $z_{d}$ is 0.3 for individual $i_{5}$, while at $z_{a}$ it is 0.1 for $i_{3}$. In other words, $z_{d}$ is preferred since the first component of $\pi\left(z_{d}\right)$ is greater than the first component of $\pi\left(z_{a}\right)$. Thus, $z_{a}$ is ignored and we continue analyzing the other three vectors. Using the same arguments $z_{d}$ is also 
ignored since the first component of $\pi\left(z_{d}\right)$ is lower than $\pi\left(z_{c}\right)$. The two remaining vectors $z_{b}$ and $z_{c}$ have identical first and second components but the third component of $\pi\left(z_{c}\right)$ is greater than $\pi\left(z_{b}\right)$, therefore $z_{b}$ is eliminated and the lexicographic maximin solution for this example is given by $z_{c}$. Summarizing, $\pi\left(z_{c}\right) \succ \pi\left(z_{b}\right) \succ \pi\left(z_{d}\right) \succ \pi\left(z_{a}\right) \Rightarrow z_{c}$ is the lexicographic maximin objective vector, and $c$ the lexicographic maximin solution.

We presented in this example a simplified scenario where the set of feasible allocations $\mathcal{X}$ is small and finite. The purpose here is just to illustrate the preference relation $\succ$ defined over the objective set, and also the order that should be followed in the search for the lexicographic maximin solution. It is important to notice, however, that in our problem the set of feasible allocations $\mathcal{X}$ is infinite and obtained from (1).

\section{Appendix B. Existence of feasible point $\boldsymbol{x}^{e}$}

Let us first make the general assumptions:

(i) there is no disconnected node in the network, i.e. from a given node it is possible to reach any other network node;

(ii) all links have capacities greater than zero: $\forall l \in \mathcal{L}, C_{l} \geq C>0$;

(iii) all utility functions are continuous and monotone with $u(\mathbf{0}, j)=0, \forall j \in \mathcal{N}$.

Let us now pose the following problem:

$$
\lambda^{*}=\max _{\boldsymbol{x} \in \mathcal{X}}\left\{\lambda \mid \sum_{k=1}^{\left|P_{j}\right|} x_{j k}=\lambda ; \forall j \in \mathcal{N}\right\}
$$

From (i) and (ii) it follows that at the solution $\boldsymbol{x}^{*}$ of problem (30) we have $\lambda^{*}>0$. From (iii) it follows that $u\left(\boldsymbol{x}^{*}, j\right)>0, \forall j \in \mathcal{N}$. Let $h>0$ be such that $h=\min _{j \in \mathcal{N}}\left\{u\left(\boldsymbol{x}^{*}, j\right)\right\}$. Since there is no restriction to reduce commodity flows, provided non-negativity is respected, for all commodity flows $i$ such that $u\left(\boldsymbol{x}^{*}, i\right)>h$ it is possible to reduce such flows until their utilities go down to $h$. In summary,

- all commodity flows $j$ such that $u\left(\boldsymbol{x}^{*}, j\right)=h$ are kept unchanged

- any flow $i$ such that $u\left(\boldsymbol{x}^{*}, i\right)>h$ can be reduced until its utility reaches $h$

Hence, there is a point $\boldsymbol{x}^{e}$ such that $u\left(\boldsymbol{x}^{e}, j\right)=h, \forall j \in \mathcal{N}$. 


\section{Acknowledgments}

The first author would like to acknowledge the support of the $\mathrm{CNPq}$ (Conselho Nacional de Desenvolvimento Cientifico e Tecnológico, Brazilian Government), which sponsored this research under grant no. 200049/99-2 during his stay at Imperial College London. The author also thanks all the support received from the Military Institute of Engineering (Instituto Militar de Engenharia - IME). Finally, the authors are in debt with the anonymous referees for their most valuable comments, which contributed to improve the quality of this paper.

\section{References}

Ahuja R K, et al. Network flows: theory, algorithms, and applications. Prentice-Hall Inc; 1993.

Awerbuch B., Shavitt Y. Converging to approximated max-min flow fairness in logarithmic time. IEEE INFOCOM 1998;3;1350-1357.

Berger T. Rate distortion theory. Prentice-Hall Inc; 1971.

Bertsekas D, Gallager R. Data networks. Prentice-Hall Inc; 1992.

Bonald T., Massoulie L. Impact of fairness on internet performance. ACM SIGMETRICS Performance Evaluation Review 2001;29;82-91.

Breslau L, Shenker S. Best-effort versus reservations: a simple comparative analysis. ACM Computer Communications Review 1998;20; 793-801.

Cao Z., Zegura E.W. Utility max-min: an application-oriented bandwidth allocation scheme. IEEE INFOCOM 1999;2;793-801.

Castro J. A specialized interior-point algorithm for multicommodity network flows. SIAM Journal on Optimization 2000;10;3; 852-877.

Castro J. Computational experience with a parallel implementation of an interior-point algorithm for multicommodity network flows. In: System Modeling and Optimization, Powell M and Scholtes S (Eds). Kluwer; 2000. p. 75-95.

Chen M. Individual monotonicity and the leximin solution. Economic Theory 2000;15; 353-365.

Denda R., et al. The fairness challenge in computer networks. Lecture Notes in Computer Science 2000;1922;208-220.

Diot C., et al. Multimedia applications should be adaptive. IEEE HPCS 1995. 
Gevros $\mathrm{P}$ et al. Congestion control mechanisms and the best-effort service model. IEEE Network 2001;15; 16-26.

Hahne E.L. Round-robin scheduling for max-min fairness in data networks. IEEE Journal on Selected Areas in Communications 1991;9:7; 1024-1039.

Kelly FP et al. Rate control for communication networks: shadow prices, proportional fairness and stability. Journal of the Operational Research Society 1998;49; 237-252.

Kelly F.P., et al. Notes on effective bandwidths - Stochastic networks: theory and applications. Oxford University Press, 1996.

Lee H W, Chong S. A distributed utility max-min flow control algorithm. Computer Networks - Elsevier 2005;50:11; 1816-1830.

Liao R, Campbell A T. A utility-based approach for quantitative adaptation in wireless packet networks. Wireless Networks 2001;7:5; 541-557.

Luce R D, Raiffa H. Games and decisions: introduction and crictical survey. Dover Publications; 1989.

Mas-Colell A, et al. Microeconomic theory. Oxford University Press; 1995.

Miettinen K M. Nonlinear multiobjective optimization. Kluwer Academic Publishers; 1998.

Pourkarimi L., Zarepisheh M. A dual-based algorithm for solving lexicographic multiple objective programs. European Journal of Operational Research 2007;176:3; 1348-1356.

Ogryczak W., Wierzbicki A. On multicriteria approaches to bandwidth allocation. Control and Cybernetics 2004;33; 427-448.

Rawls J. A theory of justice. Oxford University Press; 1999.

Richards A, et al. Mapping user level qos from a single parameter. IFIP/IEEE International Conference on Multimedia Networks and Services, 1998.

Roberts K. Interpersonal comparability and social choice theory. Review of Economic Studies 1980;47:2; 421-439.

Salles R M, Barria J A. Fair bandwidth allocation for the integration of adaptive and non-adaptive applications. Springer LNCS 2004;3126; 1-12.

Sen A K. Collective choice and social welfare. North-Holland Publishing; 1995.

Sen A K. The possibility of social choice. The American Economic Review 1999;89:3; 
349-378.

Shahrokhi F, Matula D W. The maximum concurrent flow problem. Journal of the ACM 1990;37:2; 318-334.

Shenker S. Fundamental design issues for the future internet. IEEE Journal on Selected Areas in Communications 1995;13: 7; 1176-1188.

Stein C. Approximation algorithms for multicommodity flow and shop scheduling problems. PhD Thesis: Massachusetts Institute of Technology, Cambridge - USA; 1992.

Tomaszewski A. A polynomial algorithm for solving a general max-min fairness problem. European Transactions on Telecommunications 2005;16;233-240.

Tsai W.K., Kim Y. Re-examining maxmin protocols: a fundamental study on convergence, complexity, variations, and performance. IEEE INFOCOM 1999; 811-818.

Volgenant A. Solving some lexicographic multi-objective combinatorial problems. European Journal of Operational Research 2002; 139; 578-584. 